\title{
Sociodemographic Characteristics Associated with Pretreatment Weight Change in a U.S. Military Behavioral Weight Loss Intervention
}

\author{
Margaret C. Fahey, MA ${ }^{\mathrm{a}}$, Robert C. Klesges, PhD ${ }^{\mathrm{b}, \mathrm{c}}$, Mehmet Kocak, PhD ${ }^{\mathrm{b}}$, Gerald W. \\ Talcott, PhD ${ }^{\text {,c }}$, Rebecca A. Krukowski, PhD \\ aThe University of Memphis, Department of Psychology, 400 Innovation Drive, Memphis, TN, \\ USA, 38111 \\ ${ }^{b}$ Department of Preventive Medicine, University of Tennessee Health Science Center, $66 \mathrm{~N}$ \\ Pauline Street Memphis, TN, USA, 38105 \\ 'University of Virginia, Department of Public Health Sciences, School of Medicine, 1215 Lee \\ Street, Charlottesville, VA, USA, 22908
}

Before beginning a behavioral weight loss intervention, between the initial screening visit (SV) and first baseline visit (BV), many individuals experience significant pretreatment weight change (i.e., > 1.15\% weight gain or > $1.15 \%$ weight loss) (Kerrigan et al., 2016; Tronieri et al., 2018; West, Harvey-Berino, Krukowski, Skelly, 2011). Avoiding gaining or losing at least $1.15 \%$ of one's weight has been used to define weight stability during the pretreatment period in previous studies (Kerrigan et al., 2016; Tronieri et al., 2018; West et al., 2011), because it is approximately half of what defines weight maintenance over a longer 6-month period (Wing, Tate, Gorin, Raynor, Fava, 2006). Thus, a 1.15\% weight stability criterion allows for some fluctuation, but indicates that an individual has maintained his or her body weight during this shorter time (i.e., on average between 39 and 50 days in previous studies) (Kerrigan et al., 2016; Tronieri et al., 2018; West et al., 2011).

Importantly, pretreatment weight changes have been associated with treatment outcomes (West et al., 2011). Specifically, one study found that weight loss during this waiting period was associated with greater weight loss success at the end of treatment, as well as better treatment engagement (e.g., more frequent self-monitoring) (West et al., 2011). Two other studies failed to find a relationship between pretreatment weight change and treatment outcomes (i.e., weight loss, stability, or gain); however, they observed that treatment outcomes differed depending on whether the participant's final treatment weight was compared to their SV or BV "starting" weight (Kerrigan et al., 2016; Tronieri et al., 2018). Thus, this limited research (Kerrigan et al., 2016; Tronieri et al., 2018; West et al., 2011), suggests that the pretreatment period may be relevant to an individual's overall engagement and success in a behavioral weight loss intervention.

Corresponding Author: Margaret C. Fahey; 33 N Rembert Street \#4 Memphis, TN 38104; mcfahey @memphis.edu. Availability of data and materials. Data is available upon request. 
Yet, these previous studies observed pretreatment weight change in relationship to relatively short-term treatment outcomes (i.e., 14 weeks and six months) (Kerrigan et al., 2016; Tronieri et al., 2018; West et al., 2011). In addition, previous samples were primarily female (i.e., 78.9\% - 93.7\%) (Kerrigan et al., 2016; Tronieri et al., 2018; West et al., 2011), two were mostly White/Caucasian (i.e., 65.8\% and 75.8\%) (Kerrigan et al., 2016; West et al., 2011) and one most Black (71.3\%) (Tronieri et al., 2018). None of the previous studies provided data on ethnic composition of the sample (e.g., Hispanic). Given that pretreatment weight fluctuations are likely common (Kerrigan et al., 2016; Tronieri et al., 2018; West et al., 2011), it will be important to explore potential sociodemographic factors that influence the relationship between early weight changes and intervention outcomes among racially, ethnically, and gender diverse samples. Further, to our knowledge, no previous study observed this pretreatment period in a behavioral weight loss intervention among a military sample. Given that rates of overweight and obesity in the U.S. military are high (60.8\%) (Tanofsky-Kraff et al., 2013), research aimed to understand the pretreatment waiting period is important to better inform future weight loss trials in both military and civilian populations.

Thus, the aim of the current study is to analyze data from a 12-month behavioral weight loss intervention in a U.S. military population by measuring pretreatment weight change in relationship to mid and long-term weight outcomes (i.e., four and 12-months). In addition, the study aims to explore sociodemographic differences in pretreatment weight change, as well as potential differences in its relationship to treatment success among this diverse sample of active duty personnel. Finally, this study will examine differences in pretreatment weight change in relationship to treatment self-monitoring adherence.

\section{Methods}

Procedure

The study was approved by the Institutional Review Board of the Wilford Hall Ambulatory Surgical Center in San Antonio, Texas and acknowledged by the Institutional Review Board at the University of Tennessee Health Science Center. Active duty military personnel [ $>18$ years of age, body mass index $(\mathrm{BMI})>25.0 \mathrm{~kg} / \mathrm{m} 2)$ ] were recruited from Joint Base San Antonio. Participants were required to have reliable phone and computer access and have at least one more year left in their duty assignment (to facilitate follow-up). They also had to complete one week of daily dietary and physical activity self-monitoring and obtain a letter from their health care provider approving participation in the study. Exclusion criteria included medical or psychiatric conditions that would impact the participant's ability to participate in a weight management intervention, recent substantial weight loss, current or recent pregnancy, taking medication that affects weight, or more than one failed military fitness test.

At the initial screening visit (SV), informed consent was obtained, and participants were asked to self-monitor diet and physical activity for a week and receive clearance from a healthcare provider. Subsequently, at the baseline visit (BV), participants were randomized at the individual level using a computerized block design to receive either a counselorinitiated (CI) or self-paced (SP) weight-loss intervention. Personalized weight loss, calorie, 
and exercise goals were the same across conditions and based on the Look AHEAD intensive lifestyle intervention (Look Ahead Group, 2006). Each condition received a 12month manualized behavioral weight loss program. However, the SP condition required more self-initiation to receive services (e.g., request intervention session or self-monitoring feedback).

Across both conditions, participants were asked to monitor food intake and physical activity using the Lose It ${ }^{\mathrm{TM}}$ application or website. Participants were also encouraged to self-weigh daily using the Body Trace ${ }^{\mathrm{TM}}$ electronic scales and to replace two meals and a snack with meal replacements (e.g., Healthy Choice TM, Smart Pop TM popcorn) for the first four-months to achieve calorie and fat goals. Up to 8-months, participants were encouraged to replace one meal a day with meal replacements and transition to conventional meals between months 9 and 12. With participant permission, self-monitoring data was accessed by intervention counselors to facilitate feedback. Further details on study design and outcomes have been previously reported (Krukowski et al., 2015; Krukowski et al., 2018).

\section{Measures}

Height (in centimeters) was measured using a wall-mounted stadiometer at SV and BV. At the SV, BV, four-month and 12-month visits, weight measurements were collected on a calibrated digital scale (Tanita BWB-800S). BMI (overweight: $25.0-29.9 \mathrm{~kg} / \mathrm{m}^{2}$ or obese: > $30 \mathrm{~kg} / \mathrm{m}^{2}$ ) was calculated from BV measurements. If clinic weight was missing for followups, BodyTrace ${ }^{\mathrm{TM}}$ weights closest ( \pm 30 days) to these time points were used, based on the strong concordance between in-clinic and e-scale weight measurements (Ross \& Wing, 2016; Pebley et al., 2019) After this step, missing weights at 4-months $(n=13)$ and 12months $(n=42)$ were imputed as baseline weight carried forward as a conservative estimate of these participants' weight loss outcomes and consistent methodology with West and colleagues (West et al., 2011). Participants identified gender (i.e., male, female), age (i.e., $<30$ years, 30-40 years, >40 years), ethnicity [i.e., (non-Hispanic/Latino, Hispanic/Latino)], and race [i.e., Caucasian, African American, and Other (i.e., race categories that were infrequently reported)] at SV. From SV to BV, individuals were categorized as those who lost weight $(>1.15 \%$ decrease), remained weight stable, and gained weight $(>1.15 \%$ increase) similar to previous studies (Kerrigan et al., 2016; Tronieri et al., 2018; West et al., 2011). This criterion is half of $2.3 \%$ used in research defining weight maintenance (Wing et al., 2006).

\section{Results}

Participant characteristics are provided in Table 1. The SV and BV were between 6 to 67 days apart $[$ mean $(M)=14.6$, standard deviation $(\mathrm{SD})=8.3]$, and participants experienced a median 0.2 kilogram $(\mathrm{kg})$ weight decrease $(\mathrm{M}=-0.6 \mathrm{~kg}, \mathrm{SD}=1.71)$, corresponding to a median $0.07 \%$ loss $(\mathrm{M}=-0.08, \mathrm{SD}=0.92)$. Most $(59.4 \%)$ participants were categorized as weight stable, $24.4 \%$ as having lost weight and $16.1 \%$ as having gained weight. Using Wilcoxon Mann-Whitney and Kruskal-Wallis tests, there were no significant differences in gender, BMI, ethnicity, age, military rank, and intervention condition (i.e., CI versus SP) between weight change categories (Table 2). Individuals identified as African American and 
classified as Other race were more likely to gain weight (28\% and $23.7 \%$, respectively) compared to those identified as Caucasian $(11.5 \%)(p=0.037)$. Using Spearman's rank correlation, fewer days between $\mathrm{SV}$ and BV was associated with the weight stable category $(p<0.001)$.

Step-wise regression models examined pretreatment weight change categories in relationship to treatment outcome (i.e., percent weight change from BV to four-months and BV to 12months, percent weight change from SV to four-months and SV to 12-months) after controlling for significant demographic covariates. Weight change category did not independently predict four-month outcome from BV $(p=.29)$. However, an interaction of age and pretreatment weight change $(p=0.038)$ indicated that for individuals who gained weight pretreatment, each one-year increase in age was predictive of $0.28 \%(\mathrm{SE}=0.10 \%)$ increased 4-month weight loss. Pretreatment weight change category also did not independently predict $\mathrm{BV}$ to 12 -month outcome $(p=.63)$. A non-significant interaction trend of age and weight change category $(p=0.051)$ indicated that for those who gained weight pretreatment, each one-year increase in age was predictive of $0.36 \%(\mathrm{SE}=0.12 \%)$ increased weight loss at 12-months.

After adjusting for significant covariates (i.e., gender, race, education, military grade, enrollment season, age, BMI, SV weight), weight change category predicted SV to fourmonth outcome $(p=.0005)$. Those who lost pretreatment weight were more likely to lose weight at four-months $(\mathrm{M}=-4.91 \mathrm{~kg})$ compared to those who remained weight stable $(\mathrm{M}=$ $-2.32 \mathrm{~kg}$ ) or gained weight $(\mathrm{M}=-1.69 \mathrm{~kg})$. Weight change category did not predict $\mathrm{SV}$ to 12 -month outcome $(\mathrm{p}=.19)$. Examining the relationship between pretreatment weight change and intervention adherence, Wilcoxon Mann-Whitney and Kruskal-Wallis tests revealed that pretreatment weight change category was correlated with the number of meal replacement meals used at four-months $(\mathrm{p}=.025)$ and 12-months $(\mathrm{p}=.012)$. Specifically, those who lost pretreatment weight used more meal replacements during the intervention (M $=51.3$ by four-months, $\mathrm{M}=85.3$ by 12-months) compared to those who gained weight $(\mathrm{M}=$ 31.3 by four-months, $\mathrm{M}=48.1$ by 12 -months) or remained weight stable ( $\mathrm{M}=32.1$ by fourmonths, $\mathrm{M}=46.3$ by 12 -months). However, pretreatment weight change categories were not significantly related to self-weighing frequency, dietary, or exercise self-monitoring during the intervention.

\section{Discussion}

Similar to two previous studies, most individuals in the current sample remained within $1.15 \%$ of their weight from SV to BV (Kerrigan et al., 2016; West et al., 2011). However, a much smaller percentage of participants gained weight pretreatment compared to all previous findings (16\% versus 23\%, 29.7\%, and 48.9\%) (Kerrigan et al., 2016; Tronieri et al., 2018; West et al., 2011). Importantly, only West and colleagues (2011), like the current protocol, required dietary and exercise self-monitoring before BV. Participants in West and colleagues (2011) experienced weight gain at a more similar rate (23\%) (West et al., 2011) as this study (16\%), suggesting that self-monitoring between SV and BV, perhaps, protected some individuals from experiencing significant weight gain. 
The current study had fewer days, on average, during the pretreatment period compared to the previous studies ( $M=14.6$ versus 39.4, 42.7, and 50) (Kerrigan et al., 2016; Tronieri et al., 2018; West et al., 2011). Because there was a shorter window between SV to BV due to individual randomization, perhaps, fewer participants had the opportunity to gain weight. And, not surprisingly, fewer days between SV and BV was associated with weight stability. However, only one previous study found a similar association between weight change and pretreatment length of time (Tronieri et al., 2018). Yet, despite the short time window in the current study, almost half (40.5\%) of individuals still experienced significant weight fluctuations. Current results are an extension of previous findings (Kerrigan et al., 2016; Tronieri et al., 2018; West et al., 2011), suggesting that some participants start to make significant behavioral changes during pretreatment period of weight loss interventions and other participants may use the time between SV and BV to indulge in foods that they believe will be restricted once they begin the intervention. Thus, recommending that participants continue to engage in self-regulation behaviors (e.g., self-monitoring, self-weighing) beyond the potential behavioral run-in might provide support to those more vulnerable to early weight gains.

For example, individuals identified or classified as African American and Other race were more likely to gain weight during the pretreatment period compared to those identified as Caucasian, in this more racially and ethnically diverse sample (i.e., 20\% African American, 15\% Other races; $23 \%$ Hispanic/Latino). This racial difference was contrary to all previous findings suggesting no demographic differences in pretreatment changes (Kerrigan et al., 2016; Tronieri et al., 2018; West et al., 2011), even in the previous sample that was predominately Black (Tronieri, 2018). Although there were no racial differences in the impact of these gains on eventual treatment outcome, future weight loss interventions might need to account for both SV and BV starting weights when examining racial differences in treatment efficacy, particularly if these findings are replicated in future research. Although this study was more diverse by gender (51\% female) compared to previous studies (Kerrigan et al., 2016; Tronieri et al., 2018; West et al., 2011), no gender differences were found in pretreatment weight change.

Consistent with two previous studies (Kerrigan et al., 2016; Tronieri et al., 2018), pretreatment weight change was related to treatment outcomes when weight loss success was measured starting with the screening visit. In the current study, those who lost pretreatment weight were more likely to lose weight from SV to 4-months. However, the current study observed outcomes over a longer time compared to previous studies (Kerrigan et al., 2016; Tronieri et al., 2018; West et al., 2011) and found that pretreatment weight change was not independently related to outcomes at 12-months when starting from either $\mathrm{SV}$ or BV weghts. These findings indicate that pretreatment weight changes may be more influential to success earlier in treatment, especially when the pretreatment period is included when measuring treatment outcomes.

However, in the current study, sociodemographic differences influenced the relationship between the pretreatment period and outcomes. Among individuals who gained weight pretreatment, younger adults were more vulnerable to less weight loss compared to older individuals. Importantly, younger personnel in the current sample were also less likely to be 
retained for data collection, as well as experienced less weight loss overall (Krukowski et al., 2018). It may be that weight gain before an intervention is more predictive of poor outcomes for younger participants. Perhaps, young adults are more likely to feel discouraged after experiencing pretreatment gains and could benefit from intervention support during this period (Kerrigan et al., 2016). Notably, these age group differences might be unique given that this sample was slightly younger than previous samples (Kerrigan et al., 2016; West et al., 2011; Tronieri et al., 2018) since unfortunately, behavioral weight loss trials often underrepresent individuals between 18-35 years of age (Gokee-LaRose et al., 2009; Griffin, O’Connor, Rooney, Steinbeck, 2013).

Surprisingly, pretreatment weight change was not related to most weight loss behaviors within the intervention (i.e., self-weighing, dietary, or exercise self-monitoring frequency), dissimilarly from past findings (West et al., 2011). However, pretreatment weight loss was associated with replacing a higher frequency of meals and snacks with low calorie and lowfat meal replacements. Findings indicate that perhaps, pretreatment behaviors are predictive of engagement with some, but not all, intervention protocols. If future trials include a behavioral run-in during the pretreatment period (e.g., self-weighing, diet or physical activity monitoring), researchers might consider measuring self-monitoring frequency and qualitative feedback about these behaviors before beginning an intervention. Perhaps, participant success and/or perceptions of pretreatment protocol might influence their engagement with other intervention protocol throughout the weight loss trial.

Potential limits to generalizability in the current sample of active duty military personnel should be acknowledged. This sample has access to additional weight loss resources (e.g., free fitness centers, healthcare) as well as participates in annually required fitness tests. Although the prevalence of overweight and obesity in the U.S. military is high (60.8\%) (Tanofsky-Kraff et al., 2013) the current sample had lower starting weights compared to previous studies (Krukowski et al., 2018). Yet, enrollment in this setting facilitated the inclusion of a younger and a more diverse sample in race, ethnicity, and gender compared to previous weight loss research (Gokee-LaRose et al., 2009; Griffin et al., 2013; Pagoto et al., 2012). Finally, although there was no difference in pretreatment weight change and later randomization to either the $\mathrm{CI}$ or SP condition, distribution of pretreatment weight change categories might not have been perfectly randomized across the two treatment conditions (Tronieri et al., 2018).

\section{Conclusions}

Due to limited and conflicting literature, it is critical to explore the relevance of pretreatment weight change in relationship to treatment success. This study extends previous findings that indicate many adults experience significant weight change within a relatively short time period before beginning a weight loss intervention (Kerrigan et al., 2016; Tronieri et al., 2018; West et al., 2011). Perhaps, behavioral weight loss interventions should account for the potential influence of this period on treatment outcomes. For example, during screening, participants could be encouraged to either begin practicing weight loss behaviors or remain weight stable. In addition to dietary and physical self-monitoring as a behavioral run-in before BV, future interventions could also ask participants to begin daily self-weighing. Self- 
weighing before the intervention might facilitate weight stability, as well as give participants a "taste" of this behavior which is now commonly integrated in weight loss interventions (Shieh, Knisley, Clark, Carpenter, 2016; Steinberg et al., 2013). In addition, as noted by Kerrigan and colleagues (Kerrigan et al., 2016), it will be important for researchers to include multiple "starting points" (i.e., SV, BV, first week of treatment) when observing intervention outcomes, given the commonality of early treatment weight fluctuations. Findings also suggest that for some populations (i.e., younger adults), pretreatment weight change is more predictive of treatment outcomes. Finally, future studies should observe pretreatment weight change in relationship to weight loss outcomes long-term, following up with participants even after the conclusion of the weight loss trial.

\section{Acknowledgments.}

The research represents a Collaborative Research and Development Agreement with the United States Air Force (CRADA \#13-168-SG-C13001). The opinions expressed in this document are solely those of the authors and do not represent an endorsement by or the views of the United States Air Force, the Department of Defense, or the United States Government. Finally, we would like to thank the participants and the research team for their dedication to the research. All authors have read and approved this manuscript.

Funding. The study was funded by the National Institute of Diabetes and Digestive and Kidney Diseases (RO1 DK097158) of the National Institutes of Health, with the title of "Dissemination of the Look Ahead Weight Management Treatment in the Military," Robert Klesges and Rebecca Krukowski, Principal Investigators. The trial is registered on clinicaltrials.gov (NCT 02063178).

\section{References}

Gokee-LaRose J, Gorin AA, Raynor HA, Laska MN, Jeffery RW, Levy RL, \& Wing RR (2009). Are standard behavioral weight loss programs effective for young adults? International Journal of Obesity, 33(12), 1374. [PubMed: 19786967]

Griffin HJ, O’Connor HT, Rooney KB, \& Steinbeck KS (2013). Effectiveness of strategies for recruiting overweight and obese Generation $\mathrm{Y}$ women to a clinical weight management trial. Asia Pacific journal of clinical nutrition, 22(2), 235. [PubMed: 23635367]

Kerrigan SG, Schaumberg K, Kase C, Gaspar M, Forman E, \& Butryn ML (2016). From last supper to self-initiated weight loss: Pretreatment weight change may be more important than previously thought. Obesity, 24(4), 843-849. [PubMed: 26898653]

Krukowski RA, Hare ME, Talcott GW, Johnson KC, Richey PA, Kocak M, ... \& Klesges RC (2015). Dissemination of the Look AHEAD intensive lifestyle intervention in the United States Air Force: study rationale, design and methods. Contemporary clinical trials, 40, 232-239. [PubMed: 25545025]

Krukowski RA, Hare ME, Talcott GW, Gladney LA, Johnson KC, Richey PA, ... \& Klesges RC (2018). Dissemination of the look AHEAD intensive lifestyle intervention in the United States military: a randomized controlled trial. Obesity, 26(10), 1558-1565. [PubMed: 30277030]

Look AHEAD Research Group. (2006). The Look AHEAD study: a description of the lifestyle intervention and the evidence supporting it. Obesity, 14(5), 737-752. [PubMed: 16855180]

Pagoto SL, Schneider KL, Oleski JL, Luciani JM, Bodenlos JS, \& Whited MC (2012). Male inclusion in randomized controlled trials of lifestyle weight loss interventions. Obesity, 20(6), 1234-1239. [PubMed: 21633403]

Pebley K, Klesges RC, Talcott GW, Kocak M, \& Krukowski RA (2019). Measurement Equivalence of E-Scale and In-Person Clinic Weights. Obesity.

Ross KM, \& Wing RR (2016). Concordance of in-home 'smart'scale measurement with body weight measured in-person. Obesity science \& practice, 2(2), 224-228. [PubMed: 27499884]

Shieh C, Knisely MR, Clark D, \& Carpenter JS (2016). Self-weighing in weight management interventions: a systematic review of literature. Obesity research \& clinical practice, 10(5), 493519. [PubMed: 26896865] 
Steinberg DM, Tate DF, Bennett GG, Ennett S, Samuel-Hodge C, \& Ward DS (2013). The efficacy of a daily self-weighing weight loss intervention using smart scales and e-mail. Obesity, 21(9), 17891797. [PubMed: 23512320]

Tanofsky-Kraff M, Sbrocco T, Theim KR, Cohen LA, Mackey ER, Stice E, ... \& Stephens MB (2013). Obesity and the US military family. Obesity, 21(11), 2205-2220. [PubMed: 23836452]

Tronieri JS, Wadden TA, Alfaris N, Chao AM, Alamuddin N, Berkowitz RI, \& Pearl RL (2018). "Last Supper" Predicts Greater Weight Loss Early in Obesity Treatment, but Not Enough to Offset Initial Gains. Frontiers in psychology, 9.

West DS, Harvey-Berino J, Krukowski RA, \& Skelly JM (2011). Pretreatment weight change is associated with obesity treatment outcomes. Obesity, 19(9), 1791-1795. [PubMed: 21331064]

Wing RR, Tate DF, Gorin AA, Raynor HA, \& Fava JL (2006). A self-regulation program for maintenance of weight loss. New England Journal of Medicine, 355(15), 1563-1571. [PubMed: 17035649] 
Table 1.

\begin{tabular}{|c|c|c|}
\hline \multirow{7}{*}{ 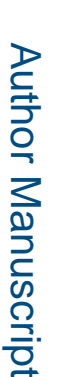 } & \multicolumn{2}{|l|}{ Study Sample } \\
\hline & Demographic Characteristics & $(\%)$ \\
\hline & \multicolumn{2}{|l|}{ Gender } \\
\hline & Male & $122(49 \%)$ \\
\hline & Female & $126(51 \%)$ \\
\hline & \multicolumn{2}{|l|}{ Age } \\
\hline & $<30$ years & $66(27 \%)$ \\
\hline & $30-40$ years & $123(50 \%)$ \\
\hline & $>40$ years & $59(24 \%)$ \\
\hline & \multicolumn{2}{|l|}{$\underline{\text { Race }}$} \\
\hline & African American & $49(20 \%)$ \\
\hline & Caucasian & $163(66 \%)$ \\
\hline 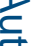 & Other & $36(15 \%)$ \\
\hline$\underline{0}$ & \multicolumn{2}{|l|}{ Ethnicity } \\
\hline$z$ & Non-Hispanic/Latino & $192(77 \%)$ \\
\hline$\coprod^{\beth}$ & Hispanic/Latino & $56(23 \%)$ \\
\hline$\frac{\bar{c}}{\omega}$ & \multicolumn{2}{|l|}{ BMI Category } \\
\hline 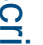 & Normal & 1 \\
\hline & Overweight & $139(55 \%)$ \\
\hline & Obese & $107(43 \%)$ \\
\hline
\end{tabular}

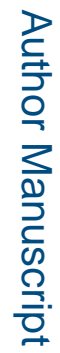

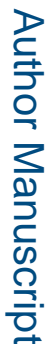

Mil Behav Health. Author manuscript; available in PMC 2021 January 01. 
Table 2.

Participant Characteristics by Pre-Treatment Weight Change Categories

\begin{tabular}{|c|c|c|c|c|}
\hline & \multicolumn{3}{|c|}{ Pretreatment Weight Change } & \multirow[t]{2}{*}{ P-Value } \\
\hline & Loss & Stable & Gain & \\
\hline Gender & & & & .123 \\
\hline Male (\%) & 45.16 & 53.64 & 36.59 & \\
\hline Female $(\%)$ & 54.84 & 46.36 & 63.41 & \\
\hline Race & & & & .024 \\
\hline African American (\%) & 12.90 & 18.54 & 34.15 & \\
\hline Caucasian (\%) & 74.19 & 67.55 & 43.90 & \\
\hline Other $(\%)$ & 12.90 & 13.91 & 21.95 & \\
\hline \multicolumn{5}{|l|}{ Ethnicity } \\
\hline Non-Hispanic/Latino (\%) & 72.58 & 78.15 & 80.49 & .58 \\
\hline Hispanic/Latino (\%) & 27.42 & 21.85 & 19.51 & \\
\hline Education & & & & .28 \\
\hline Less than college (\%) & 41.94 & 52.32 & 56.10 & \\
\hline College or higher (\%) & 58.06 & 47.68 & 43.90 & \\
\hline Military Grade & & & & .119 \\
\hline E1-E4 (\%) & 14.52 & 15.23 & 4.88 & \\
\hline E5-E6 (\%) & 38.71 & 45.70 & 43.90 & \\
\hline E7-E9 $(\%)$ & 20.97 & 16.56 & 36.59 & \\
\hline O1-O3 & 11.29 & 5.96 & 2.44 & \\
\hline O4-O6 (\%) & 14.52 & 15.89 & 12.20 & \\
\hline Age (years) $M(S D)$ & $35.10(8.10)$ & $34.0(7.40)$ & $35.2(6.50)$ & .72 \\
\hline Days from SV to BV $M(S D)$ & $15.50(10.20)$ & $11.0(13.50)$ & $15.00(17.20)$ & .0014 \\
\hline
\end{tabular}

Note. Military ranking; Enlisted (E) categories: E1-E4 (enlisted), E5-E6 (non-commissioned officers), E7-E9 (senior non-commissioned officers) and 2 Officer categories (O): O1-O3 (Company Grade Officer) and O4-O6 (Field Grade Officer); Mean (MD); Standard deviation (SD) 